\title{
The rank gradient from a combinatorial viewpoint
}

\author{
Miklós Abért ${ }^{1}$, Andrei Jaikin-Zapirain ${ }^{2}$ and Nikolay Nikolov
}

Fritz, thank you for the conversations!

\begin{abstract}
This paper investigates the asymptotic behaviour of the minimal number of generators of finite index subgroups in residually finite groups. We analyze three natural classes of groups: amenable groups, groups possessing an infinite soluble normal subgroup and virtually free groups. As a tool for the amenable case we generalize Lackenby's trichotomy theorem on finitely presented groups.
\end{abstract}

Mathematics Subject Classification (2010). 20F69, 20 E06.

Keywords. Rank gradient, amalgam, amenable group, Lück approximation.

\section{Introduction}

Let $\Gamma$ be a finitely generated group. A chain in $\Gamma$ is a decreasing infinite sequence $\Gamma=\Gamma_{0}>\Gamma_{1}>\cdots$ of subgroups of finite index in $\Gamma$. The chain is normal if all $\Gamma_{n}$ are normal in $\Gamma$.

For a group $\Gamma$ let $d(\Gamma)$ denote the minimal number of generators (or rank) of $\Gamma$. For a subgroup $H \leq \Gamma$ of finite index let

$$
r(\Gamma, H)=(d(H)-1) /|\Gamma: H|,
$$

and let the rank gradient of $\Gamma$ with respect to the chain $\left(\Gamma_{n}\right)$ be defined as

$$
\operatorname{RG}\left(\Gamma,\left(\Gamma_{n}\right)\right)=\lim _{n \rightarrow \infty} r\left(\Gamma, \Gamma_{n}\right)
$$

This notion has been introduced by Lackenby [6].

In a previous paper [1] the first and third authors investigated the rank gradient using analytic tools, namely, the theory of cost. This tool is applicable only if the

\footnotetext{
${ }^{1}$ M. Abért is supported by the grants Marie Curie IEF 235545, NSF DMS 0701105 and OTKA NK72523.

${ }^{2}$ A. Jaikin-Zapirain is supported by the Spanish Ministry of Science and Innovation, the grant MTM2008-06680 and the Autonomous University of Madrid, grant CCG08-UAM/ESP-4145.
} 
chain $\left(\Gamma_{n}\right)$ is normal or more generally satisfies the Farber condition (that is, if the action of the group on the boundary of the associated coset tree is essentially free). A central problem discussed there is whether the rank gradient depends on the choice of normal chain, assuming that it approximates the group. This is still unknown.

The aim of this paper is to present some results on rank gradient that can be proved by elementary methods; that is, using just group theory and combinatorics.

Our first three results do not assume that the chain is normal. For non-normal chains they are new and stronger than what we can show using cost.

Theorem 1. Let $\Gamma$ be a finitely presented infinite amenable group and let $\left(\Gamma_{n}\right)$ be an arbitrary chain in $\Gamma$. Then $\operatorname{RG}\left(\Gamma,\left(\Gamma_{n}\right)\right)=0$.

This has been proved by Lackenby [6] for normal chains. The extension to arbitrary chains relies on a mild generalization of Lackenby's method plus a new ingredient, the concept of strong ergodicity for group actions discussed in Section 2. Note that the finite presentation assumption is necessary in Theorem 1, even for normal chains. Indeed, let $\Gamma=C_{2}$ 乙 $\mathbb{Z}$ be the lamplighter group and let $\Gamma_{n}$ be the normal subgroup such that $\Gamma / \Gamma_{n} \cong C_{2^{n}}$. Then it is easy to see that $\Gamma_{n}$ has a quotient equal to $C_{2}^{2^{n}}$ and so $\operatorname{RG}\left(\Gamma,\left(\Gamma_{n}\right)\right) \geq 1$. However, we do not know the answer to the following.

Question 2. Let $\Gamma$ be a finitely generated infinite amenable group and let $\left(\Gamma_{n}\right)$ be a chain in $\Gamma$ with trivial intersection. Is $\operatorname{RG}\left(\Gamma,\left(\Gamma_{n}\right)\right)=0$ ?

In Theorem 4 we answer this question affirmatively for groups containing an infinite soluble normal subgroup.

Theorem 1 is a corollary of the following generalization of Lackenby's theorem ([6], Theorem 1.1).

Theorem 3. Let $\Gamma$ be a finitely presented group and let $\left(\Gamma_{n}\right)$ be an arbitrary chain in $\Gamma$. Then at least one of the following holds:

1) the boundary action of $\Gamma$ with respect to $\left(\Gamma_{n}\right)$ is strongly ergodic;

2) $\operatorname{RG}\left(\Gamma,\left(\Gamma_{n}\right)\right)=0$;

3) there exists $n$ such that $\Gamma_{n}$ is a non-trivial amalgamated product.

A group $\Gamma$ is a nontrivial amalgamated product $A_{1} *_{A_{3}} A_{2}$ if the subgroup $A_{3}=$ $A_{1} \cap A_{2}$ is not equal to neither of $A_{1}$ or $A_{2}$ and has index at least 3 in one of them.

Compared to Lackenby's original theorem there are two new components in Theorem 3. First, part 3) of Lackenby's theorem allows the possibility that $\Gamma_{n}$ is an HNN extension - we can exclude that case.

Second, and more important, the strong ergodicity condition replaces property $(\tau)$. This is what allows us to prove Theorem 1 for non-normal chains. Strong ergodicity is weaker requirement in general than property $(\tau)$, it is known that for normal chains the two conditions are equivalent by work of the first author and Elek [2]. 
From now on we will concentrate on the case when the chain has trivial intersection.

Theorem 4. Assume that $\Gamma$ has an infinite soluble normal subgroup. Then $\Gamma$ has rank gradient zero with respect to any chain with trivial intersection.

Using cost, it is proved in [1] that if $\Gamma$ has an infinite normal amenable subgroup, then the rank gradient vanishes for normal chains with trivial intersection. However note that when the chain is not normal Theorem 4 does not follow from the results of [1] since the connection between cost and rank gradient established there cannot be applied.

In the second half of the paper we concentrate on the case when the chain is normal, in which case stronger results are obtained. Many of the results here were known previously, however our methods are more elementary and in particular we do not use any analytic tools.

Theorem 5. Finitely generated infinite amenable groups have rank gradient zero with respect to any normal chain with trivial intersection.

Theorem 5 follows from the following result of B. Weiss [15]. Recall that if $N$ is a subgroup of a group $\Gamma$ then a (left) transversal of $N$ in $\Gamma$ is a complete set of representatives of cosets $\{g N: g \in \Gamma\}$.

Theorem 6. Let $\Gamma$ be an amenable group generated by a finite set $S$ and let $\left(\Gamma_{n}\right)$ be a normal chain in $\Gamma$ with trivial intersection. Then for each $\varepsilon>0$ there exists $k \in \mathbb{N}$ and a transversal $T$ of $\Gamma_{k}$ in $\Gamma$ such that

$$
|T S \backslash T|<\varepsilon|T|
$$

We will provide a short proof for this result of Weiss. His original proof is a version of the Orenstein-Weiss quasitiling lemma; ours is more algebraic and may be interesting for further applications.

Now we look at the behaviour of $r\left(\Gamma, \Gamma_{n}\right)$ over chains. It turns out that virtually free groups can be characterized as those $\Gamma$ for which $r\left(\Gamma, \Gamma_{n}\right)$ stabilizes on normal chains $\left(\Gamma_{n}\right)$.

Theorem 7. Let $\Gamma$ be a finitely generated residually finite group.

i) If $\Gamma$ is virtually free and $\left(\Gamma_{n}\right)$ is a normal chain in $\Gamma$ with trivial intersection, then there exists $k$ such that $r\left(\Gamma, \Gamma_{i}\right)=r\left(\Gamma, \Gamma_{k}\right)($ for all $i \geq k)$.

ii) If $\left(\Gamma_{n}\right)$ is a chain of (not necessarily normal) subgroups of $\Gamma$ with trivial intersection such that the sequence $r\left(\Gamma, \Gamma_{n}\right)$ stabilizes, then $\Gamma$ is virtually free.

Next, we investigate the rank gradient of free products with amalgamation. For free products, we obtain the following equality. 
Proposition 8. Let $G_{1}$ and $G_{2}$ be finitely generated, residually finite groups. Let $\left(N_{i}\right)$ be a normal chain in $G_{1} \star G_{2}$ and put $N_{1, i}=N_{i} \cap G_{1}, N_{2, i}=N_{i} \cap G_{2}$. Then

$$
\operatorname{RG}\left(G_{1} \star G_{2},\left(N_{i}\right)\right)=\operatorname{RG}\left(G_{1},\left(N_{1, i}\right)\right)+\operatorname{RG}\left(G_{2},\left(N_{2, i}\right)\right)+1 .
$$

The second theorem is very general, but it only gives an inequality.

Proposition 9. Let $\Gamma$ be a residually finite group generated by two finitely generated subgroups $G_{1}$ and $G_{2}$ such that their intersection is infinite. Then

$$
\operatorname{RG}\left(\Gamma,\left(\Gamma_{n}\right)\right) \leq \operatorname{RG}\left(G_{1},\left(G_{1} \cap \Gamma_{n}\right)\right)+\operatorname{RG}\left(G_{2},\left(G_{2} \cap \Gamma_{n}\right)\right)
$$

for any normal chain $\left(\Gamma_{n}\right)$ in $\Gamma$. In particular, if $G_{1}$ and $G_{2}$ have vanishing rank gradient with respect to any normal chain then so does $\Gamma$.

Besides cost, the rank gradient is related to another important group invariant of $\Gamma$, the first $L^{2}$ Betti number $\beta_{1}^{(2)}(\Gamma)$. We have

$$
\operatorname{RG}\left(\Gamma,\left(\Gamma_{n}\right)\right) \geq \operatorname{cost}(\Gamma)-1 \geq \beta_{1}^{(2)}(\Gamma) .
$$

for any normal chain $\left(\Gamma_{n}\right)$ in $\Gamma$ with trivial intersection. In all the known cases so far these three numbers coincide.

There are important cases where the vanishing of the first $L^{2}$ Betti number is known but not the cost or the rank gradient. For instance, groups with Kazhdan property (T) have first $L^{2}$ Betti number equal to 0 (see [3]).

Conjecture 10. If $\Gamma$ has property $(\mathrm{T})$ and is infinite then $\mathrm{RG}\left(\Gamma,\left(\Gamma_{n}\right)\right)=0$ for any normal chain $\left(\Gamma_{n}\right)$ in $\Gamma$ with trivial intersection.

A group $\Gamma$ is said to be boundedly generated if it can be written as the product of finitely many of its cyclic subgroups $\Gamma=\left\langle g_{1}\right\rangle \cdot\left\langle g_{2}\right\rangle \ldots\left\langle g_{t}\right\rangle$. Examples of boundedly generated groups are arithmetic groups with the congruence subgroup property, like $\operatorname{SL}(d, \mathbb{Z})(d>2)$ see [14]. For many of these it follows from the results in [12] that they have vanishing rank gradient for any normal chain. We conjecture that in general the rank gradient of boundedly generated residually finite groups is zero. In this direction we can show the following.

Proposition 11. If $\Gamma$ is an infinite finitely presented residually finite boundedly generated group then the first $L^{2}$ Betti number of $\Gamma$ is zero.

The organization of the paper is as follows. In Section 2 we recall the Reidemeister-Schreier theorem and define coset trees and strong ergodicity. Theorems 3 and 1 are proved in Section 3. Section 4 contains the proof of Theorems 5 and 6. Theorem 4 is proved in Section 5 and Theorem 7, Proposition 8 and 9 are proved in Section 6. Finally in Section 7 we give a short proof of Lück's approximation theorem for amenable groups over arbitrary fields and prove Proposition 11. 


\section{Preliminaries}

First we recall the notion of Schreier graphs and the Reidemester-Schreier theorem. Let $\Gamma$ be a group generated by a finite set $S$ and $H$ a subgroup of finite index. Then the Schreier graph $\Delta=\Delta(\Gamma, H, S)$ for $\Gamma$ relative to $H$ with respect to $S$ is an oriented graph defined as follows:

1. The vertices of $\Delta$ are the left cosets of $H$ in $\Gamma$, that is $V(\Delta)=\{g H \mid g \in \Gamma\}$.

2. The set of edges $E(\Delta)$ is $\{(g H, s g H) \mid g \in \Gamma, s \in S\}$.

For a subset $A$ of $V(\Delta)$ we denote by $\partial A$ the set of edges that connects $A$ and its complement $A^{c}$ in $V(\Delta)$. It is clear that any path in the Schreier graph corresponds to a word in $\Gamma$ which is a product of some $s_{i}$, where $s_{i} \in S \cup S^{-1}$.

Let $T$ be a left transversal for $H$ in $G$. If $g \in G$, define by $\tilde{g}$ the unique $t \in T$ such that $g H=t H$. If $e=(g H, s g H), s \in S$, is an edge of the Schreier graph $\Delta=\Delta(\Gamma, H, S)$, then we put $T(e)=(\widetilde{s g})^{-1} s \tilde{g}$ and $T(\bar{e})=\tilde{g}^{-1} s^{-1} \widetilde{s g}=T(e)^{-1}$. It is known that $\{T(e)\}$ generate $H$.

In this paper we will work mostly with so called Schreier transversals with respect to $S$ for $H$ in $\Gamma$. To define them, fix a maximal tree $\mathcal{T}$ embedded in $\Delta$. Then for any $g \in \Gamma$ there exists a unique path from $H$ to $g H$. Let $T$ be the set of all words corresponding to these paths. It is clear that $T$ is a left transversal for $H$ in $\Gamma$. We call this transversal, the Schreier transversal with respect to $S$ corresponding to $\mathcal{T}$. Note that $T(e)=1$ if $e \in E(\mathcal{T})$.

Now, let us assume that $F$ is a finitely generated free group and $S$ is a set of its free generators. Let $H$ be a subgroup of $F$ of finite index and put $\Delta=\Delta(F, H, S)$. Let $N$ be a normal subgroup of $F$ contained in $H$ and generated (as a normal subgroup) by a finite set $R$. Thus, $F / N \cong\langle S \mid R\rangle$. Let $T$ be a right Schreier transversal for $H$ in $F$ corresponding to a maximal subtree $\mathcal{T}$ of $\Delta$. We want to write a presentation of $H / N$ using the generators $T(e)$. Take a relation of $F / N, r=s_{l} \ldots s_{1} \in R$, where $s_{i} \in S \cup S^{-1}$ and let $t \in T$. Then $r_{t}=t^{-1} r t$ is an element of $H$ and we can rewrite $r_{t}$ as a product of $l$ elements $T(e): r_{t}=T\left(e_{l}\right) \ldots T\left(e_{1}\right)$, where $e_{1}=\left(t H, s_{1} t H\right), \ldots, e_{l}=\left(s_{l-1} \ldots s_{1} t H, t H\right)$. Recall that $T(e)=1$ if $e \in$ $E(\mathcal{T})$, whence we can rewrite $r_{t}$ as product of at most $l$ elements $T(e)^{ \pm 1}$ with $e \in E(\Delta) \backslash E(\mathcal{T})$. It is a known fact that $H / N$ has the following presentation:

$$
H / N \cong\left\langle\{T(e)\}_{e \in E(\Delta) \backslash E(\mathcal{T})} \mid\left\{r_{t}\right\}_{r \in R, t \in T}\right\rangle .
$$

Now we define boundary actions with respect to a chain. Let $\left(\Gamma_{n}\right)$ be a chain in $\Gamma$. Then the coset tree $\mathfrak{T}=\mathfrak{T}\left(\Gamma,\left(\Gamma_{n}\right)\right)$ of $\Gamma$ with respect to $\left(\Gamma_{n}\right)$ is defined as follows. The vertex set of $\mathfrak{T}$ equals

$$
\mathfrak{T}=\left\{g \Gamma_{n} \mid n \geq 0, g \in \Gamma\right\}
$$

and the edge set is defined by inclusion, that is,

$$
\left(g \Gamma_{n}, h \Gamma_{m}\right) \text { is an edge in } \mathfrak{T} \text { if } m=n+1 \text { and } g \Gamma_{n} \supseteq h \Gamma_{m} .
$$


Then $\mathfrak{T}$ is a tree rooted at $\Gamma$ and every vertex of level $n$ has the same number of children, equal to the index $\left|\Gamma_{n}: \Gamma_{n+1}\right|$. The left actions of $\Gamma$ on the coset spaces $\Gamma / \Gamma_{n}$ respect the tree structure and so $\Gamma$ acts on $₹$ by automorphisms.

The boundary $\partial \mathfrak{T}$ of $\mathfrak{T}$ is defined as the set of infinite rays starting from the root. The boundary is naturally endowed with the product topology and product measure coming from the tree. More precisely, for $t=g \Gamma_{n} \in \mathfrak{T}$ let us define $\operatorname{Sh}(t) \subseteq \partial \mathfrak{T}$, the shadow of $t$ as

$$
\operatorname{Sh}(t)=\{x \in \partial \mathfrak{T} \mid t \in x\}
$$

the set of rays going through $t$. Set the base of topology on $\partial \mathfrak{T}$ to be the set of shadows and set the measure of a shadow to be

$$
\mu(\operatorname{Sh}(t))=1 /\left|\Gamma: \Gamma_{n}\right|
$$

This turns $\partial \mathfrak{T}$ into a totally disconnected compact space with a Borel probability measure $\mu$. The group $\Gamma$ acts ergodically on $\partial \mathfrak{T}$ by measure-preserving homeomorphisms; we call this action the boundary action of $\Gamma$ with respect to $\left(\Gamma_{n}\right)$. See [4] where these actions were first investigated in a measure theoretic sense.

Let $\Gamma$ act on a probability space $(X, \mu)$ by measure preserving maps. A sequence of subsets $A_{n} \subseteq X$ is almost invariant, if

$$
\lim _{n \rightarrow \infty} \mu\left(A_{n} \backslash A_{n} \gamma\right)=0 \quad \text { for all } \gamma \in \Gamma
$$

The sequence is trivial, if $\lim _{n \rightarrow \infty} \mu\left(A_{n}\right)\left(1-\mu\left(A_{n}\right)\right)=0$. We say that the action is strongly ergodic, if every almost invariant sequence is trivial.

In general, spectral gap implies strong ergodicity, but not the other way round. For a chain of subgroups $\left(\Gamma_{n}\right)$ in $\Gamma$, spectral gap is equivalent to Lubotzky's property $(\tau)$, while strong ergodicity means that large subsets of $\Gamma / \Gamma_{n}$ expand, but we do not know what happens to small subsets.

In this paper, we will use the following two results on strongly ergodic actions and amenability. The first is by Schmidt ([10], Theorem 2.4).

Theorem 12. Let $\Gamma$ be a countable amenable group acting on a standard Borel probability space by measure preserving maps. Then the action is not strongly ergodic.

The second result is from the first author and Elek [2].

Lemma 13. Let $\Gamma$ be a group generated by a finite symmetric set $S$ and let $\left(\Gamma_{n}\right)$ be a chain in $\Gamma$ such that the boundary action of $\Gamma$ with respect to $\left(\Gamma_{n}\right)$ is not strongly ergodic. Then for all $\varepsilon>0$ and $\alpha>\varepsilon$, for all sufficiently large $n$ there exists a subset $A \subseteq \Gamma / \Gamma_{n}$ such that

$$
\left|\frac{|A|}{\left|\Gamma: \Gamma_{n}\right|}-\alpha\right|<\varepsilon \quad \text { and } \quad|A S \backslash A|<\varepsilon|A| .
$$




\section{Finitely presented amenable groups}

First we explain the general strategy that we use to represent a finitely presented group as an amalgamated free product. Let $H=\left\langle S^{H} \mid R^{H}\right\rangle$ be a finite presentation of a group $H$. We assume that any generator from $S^{H}$ appears at least once in some relation from $R^{H}$. Suppose $R^{H}$ is presented as a union of two subsets $R_{1}$ and $R_{2}$. Denote by $S_{i}(i=1,2)$ the generators from $S$ which appears in the words from $R_{i}$. Put $S_{3}=S_{1} \cap S_{2}$ and $R_{3}=R_{1} \cap R_{2}$. Denote by $T_{i}(i=1,2,3)$ the group with the following presentation $\left\langle S_{i} \mid R_{i}\right\rangle$ and let $H_{i}(i=1,2,3)$ be the subgroup of $H$ generated by $S_{i}$. It is clear that $H_{i}$ is a quotient of $T_{i}$.

There are two natural homomorphisms $\phi_{1}: T_{3} \rightarrow T_{1}$ and $\phi_{2}: T_{3} \rightarrow T_{2}$ which need not be injective. Then $H$ is isomorphic to the pushout of the following diagram:

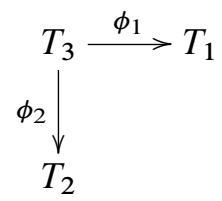

By the universal property of pushouts, $H_{1} *_{H_{3}} H_{2}$ is a homomorphic image of $H$. Hence we have the isomorphism $H \cong H_{1} *_{3} H_{2}$. Of course, in most situations this method gives us a trivial amalgamated free product (i.e. $H_{3}=H_{1}$ or $H_{3}=H_{2}$ ).

Now we give a variation of the previous construction. Suppose that $\Gamma=\langle S \mid R\rangle$ and $H$ is a subgroup of finite index $\Gamma$. Let $\Delta=\Delta(\Gamma, H, S), \mathcal{T}$ a maximal tree in $\Delta$ and $T$ the Schreier transversal for $H$ in $\Gamma$ corresponding to $\mathcal{T}$. Then $H$ has the following presentation (see (1)).

$$
H \cong\left\{\{T(e)\}_{e \in E(\Delta) \backslash E(\mathcal{T})}\left|\left\{r_{\tilde{g}}\right\}_{r \in R, \tilde{g} \in T}\right\rangle .\right.
$$

We want to use the construction described in the previous paragraph. For this we have to represent the set $R^{H}=\left\{r_{\tilde{g}}\right\}_{r \in R, \tilde{g} \in T}$ as a union of two subsets.

Let $A$ be a subset of $V(\Delta)$ and define $R^{H}(A)$ be the set of relations $r_{\tilde{g}}=$ $T\left(e_{1}\right)^{ \pm 1} \ldots T\left(e_{l}\right)^{ \pm 1}$ such that for some $1 \leq i \leq l$ one of the end points of $e_{i}$ lies in $A$. Then it is clear that $R^{H}=R_{1} \cup R_{2}$ where $R_{1}=R^{H}(A)$ and $R_{2}=R^{H}\left(A^{c}\right)$. If all generators from $S^{H}=\{T(e)\}_{e \in E(\Delta) \backslash E(\Gamma)}$ appears at least once in some relation from $R^{H}$, then using the construction from the previous paragraph we obtain the decomposition $H \cong H_{1} *_{H_{3}} H_{2}$. If a generator $T(e)$ does not appear in any relation then we add it to $S_{1}$ if $e$ connects two elements from $A$, to $S_{2}$ if $e$ connects two elements from $A^{c}$ and to $S_{1}$ and $S_{2}$ if $e$ is contained in $\partial A$. In this case we obtain again $H \cong H_{1} *_{H_{3}} H_{2}$.

There is an easy description of the generating sets $S_{i}$ of $H_{i}(i=1,2,3)$. Let $X_{1}$ consists of elements $T(e)$ such that the both end points of $e$ are in $A, X_{2}$ consists of elements $T(e)$ such that the both end points of $e$ are in $A^{c}$ and $X_{3}$ consist of elements $T(e)$ such that either $e \in \partial A$ or there exists a relation $r_{\tilde{g}}=T\left(e_{1}\right)^{ \pm 1} \ldots T\left(e_{l}\right)^{ \pm 1}$ for 
which some $e_{i} \in \partial A$ and some $e_{j}$ is equal to $e$. Then we obtain that $S_{1}=X_{1} \cup X_{3}$, $S_{2}=X_{2} \cup X_{3}$ and $S_{3}=X_{3}$.

We are ready to prove the modified trichotomy result of Lackenby.

Proof of Theorem 3. We assume that $\Gamma$ does not satisfy 1) and 2) and will show that then 3) holds. Since for $i \geq j$ we have

$$
d\left(\Gamma_{j}\right)-1 \geq \frac{d\left(\Gamma_{i}\right)-1}{\left|\Gamma_{j}: \Gamma_{i}\right|}
$$

the sequence $\left(d\left(\Gamma_{i}\right)-1\right) /\left|\Gamma: \Gamma_{i}\right|$ is non-increasing. Thus, using $\operatorname{RG}\left(\Gamma,\left(\Gamma_{n}\right)\right)>0$ and changing (if needed) $\Gamma$ by $\Gamma_{j}$ we may assume that

$$
d\left(\Gamma_{i}\right)-1 \geq \frac{3\left|\Gamma: \Gamma_{i}\right| d(\Gamma)}{4}
$$

for all $i$.

Fix a finite presentation $\Gamma=\langle S \mid R\rangle$ such that $|S|=d(\Gamma)$. Let $L$ be the sum of the lengths of elements from $R$. Since the boundary action of $\Gamma$ with respect to $\left(\Gamma_{n}\right)$ is not strongly ergodic, using Lemma 13 there exist $i$ and $A \in V\left(\Delta\left(\Gamma, \Gamma_{i}, S\right)\right)$ such that

1. $\frac{\left|\Gamma: \Gamma_{i}\right|}{4}<|A|<\frac{\left|\Gamma: \Gamma_{i}\right|}{2}$, and

2. $|\partial A|<\frac{1}{2\left(1+L^{2}\right)}|A|$.

Put $H=\Gamma_{i}$ and $\Delta=\Delta(\Gamma, H, S)$. Fix a maximal tree $\mathcal{T}$ in $\Delta$ and let $T$ be the right Schreier transversal corresponding to this tree.

Now we apply the construction described at the beginning of this section. We obtain that $\mathrm{H}$ is isomorphic to an amalgamated free product of $H_{1} *_{H_{3}} H_{2}$. We will prove that $H_{3}$ has index at least 4 in both $H_{1}$ and $H_{2}$. We use the previous notation, so $H_{1}$ is generated by $S_{1}=X_{1} \cup X_{3}, H_{2}$ is generated by $S_{2}=X_{2} \cup X_{3}$ and $H_{3}$ is generated by $S_{3}=X_{3}$.

Suppose that $H_{1}$ has index at most 3 in $H_{3}$. Then $H$ is generated by $H_{2}$ and at most one other element and so $d(H) \leq d\left(H_{2}\right)+1$. It is easy to see that

$$
\left|X_{2}\right| \leq|S|\left|A^{c}\right|-\left|A^{c}\right|+1=(d(\Gamma)-1)\left|A^{c}\right|+1<\frac{3(d(\Gamma)-1)|V(\Delta)|}{4}+1 .
$$

Let $r=s_{l} \ldots s_{1}$ be a relation of $\Gamma$ of length $l$. Note that there are at most $l|\partial A|$ different lifts $r_{\tilde{g}}=T\left(e_{1}\right)^{ \pm 1} \ldots T\left(e_{l}\right)^{ \pm 1}$ of $r$ for which some $e_{i} \in \partial A$. And also for each such relation of $H$ we have at most $l$ generators $T(e)$ of $H$ which are getting into $X_{3}$. Thus, if $\left\{l_{i}\right\}$ is the set of the lengths of the relations of $\Gamma$ (so $L=\sum l_{i}$ )), then we have that

$$
\left|S_{3}\right|=\left|X_{3}\right| \leq|\partial A|\left(1+\sum l_{i}^{2}\right) \leq|\partial A|\left(1+L^{2}\right) \leq \frac{|V(\Delta)|}{2} .
$$


Thus, we obtain that

$$
\frac{3 d(\Gamma)|V(\Delta)|}{4}+1 \leq d(H) \leq\left|X_{2}\right|+\left|X_{3}\right|+1<\frac{3(d(\Gamma)-1)|V(\Delta)|}{4}+2+\frac{|V(\Delta)|}{2} .
$$

This is a contradiction when $|V(\Delta)|>8$ and therefore $\left|H_{1}: H_{3}\right|>3$. In the same way we obtain that $\left|H_{2}: H_{3}\right|>3$.

Proof of Theorem 1. Using Theorem 13, the boundary action of $\Gamma$ with respect to $\left(\Gamma_{n}\right)$ is not strongly ergodic. Since $\Gamma$ is amenable, it is not virtually a nontrivial amalgamated product. Using Theorem 3 now, we get that 2) must hold, that is, $\operatorname{RG}\left(\Gamma,\left(\Gamma_{n}\right)\right)=0$.

\section{Normal chains in amenable groups}

In this section it will be sometimes convenient to work with multisets instead of sets. If $S$ is a multiset then $|S|$ denotes the total number of elements of $S$ counted with repetitions.

Let $\Gamma$ be a group generated by a finite multiset $S$ and $A$ a finite multisubset of $\Gamma$. The boundary of $A$ with respect to $S$ is the multiset

$$
\partial_{S}(A)=\{(a, s a) \mid a \in A, s \in S, s a \notin A\} .
$$

We say that $A$ is $\varepsilon$-invariant (with respect to $S$ ) if $\left|\partial_{S}(A)\right| \leq \varepsilon|S||A|$. Recall that $\Gamma$ is amenable if for each $n$ there exists a sequence $\left\{A_{n}\right\}$ of finite subsets of $\Gamma$ such that $A_{n}$ is $a_{n}$-invariant and $\lim _{n \rightarrow \infty} a_{n}=0$. We say that $\left\{A_{n}\right\}$ is a Følner sequence. It is easy to see that the definitions of the amenability and a Følner sequence do not depend on the generating multiset $S$.

Next we proceed with a general lemma on coverings.

Lemma 14. Let $G$ be a compact topological group with normalised Haar measure $\mu$ and let $A \subseteq G$ be a measurable subset of positive measure. For a natural number $k$ let

$$
\operatorname{cov}(A, k)=\max _{\substack{X \subseteq G \\|X|=k}} \mu(A X)
$$

where

$$
A X=\{a x \mid a \in A, x \in X\}
$$

Then

$$
\operatorname{cov}(A, k) \geq 1-(1-\mu(A))^{k} .
$$

In particular, for $k=\lceil 1 / \mu(A)\rceil$ we have

$$
\operatorname{cov}(A, k)>1-\frac{1}{\mathrm{e}}
$$


Proof. First, the definition makes sense, since the maximum is always achieved by compactness. We prove the statement using induction on $k$. For $k=1$ the statement is trivial.

Assume the lemma holds for $k-1$; this implies that there exists a subset $X \subseteq G$ of size $k-1$ such that $\mu(A X) \geq 1-(1-\mu(A))^{k-1}$. Let $B=A X$ and let us define the subset

$$
U=\{(a, g) \in G \times G \mid a \in A, a g \in B\} .
$$

Now $U$ is measurable in $G \times G$ and using Fubini's theorem we have

$$
\mu^{2}(U)=\int_{a \in A} \mu\left(a^{-1} B\right)=\mu(A) \mu(B)
$$

where $\mu^{2}$ denotes the product measure on $G \times G$. Now using Fubini's theorem from the other side gives

$$
\mu^{2}(U)=\int_{g \in G} \mu(A g \cap B) .
$$

If for all $g \in G$ we have $\mu(A g \cap B)>\mu(A) \mu(B)$ then

$$
\mu(A) \mu(B)=\int_{g \in G} \mu(A g \cap B)>\mu(A) \mu(B),
$$

a contradiction (we used $\mu(G)=1$ ). So there exists $g \in G$ such that

$$
\mu(A g \cap B) \leq \mu(A) \mu(B)
$$

which implies $\mu(A g \backslash B) \geq \mu(A)-\mu(A) \mu(B)$. Now let $X^{\prime}=X \cup\{g\}$. For this $X^{\prime}$ we have

$$
\mu\left(A X^{\prime}\right)=\mu(B)+\mu(A g \backslash B) \geq \mu(B)(1-\mu(A))+\mu(A) \geq 1-(1-\mu(A))^{k}
$$

using $\mu(B) \geq 1-(1-\mu(A))^{k-1}$. So the statement of the lemma holds.

Finally setting $k=\lceil 1 / \mu(A)\rceil$ we have

$$
\operatorname{cov}(A, k) \geq 1-(1-\mu(A))^{k} \geq 1-(1-\mu(A))^{1 / \mu(A)}>1-\frac{1}{\mathrm{e}}
$$

using $0<\mu(A) \leq 1$.

Note that for finite groups one can get a slightly better estimate using that the intersection has integer size. What we really need here is an absolute constant greater than $\frac{1}{2}$.

Proof of Theorem 6. The proof consist of two steps. First we will show that there exists a $c$-invariant transversal for some $c<1$, and then iterating the first step $k$ times we will obtain $c^{k}$-invariant transversal. 
Step 1. Let $\delta=\frac{0.1}{1.1 \cdot \mathrm{e}}$ and let $A$ be a $\delta$-invariant set with respect to $S$. Since the intersection of $\Gamma_{i}$ is trivial there exists $j \in \mathbb{N}$ such that the projections $\bar{a}=a \Gamma_{j}$ of the elements $a \in A$ in $\bar{\Gamma}=\Gamma / \Gamma_{j}$ are all different and $\frac{|\bar{\Gamma}|}{|A|}>10$. Now applying Lemma 14, we obtain that there exists a subset $X$ of $\Gamma$ of size $\left\lceil\frac{|\bar{\Gamma}|}{|A|}\right\rceil$ (in particular, $|\bar{\Gamma}| \leq|A||X| \leq|\bar{\Gamma}|+|A| \leq 1.1|\bar{\Gamma}|)$ such that the size of the set $\overline{A X}$ is at least $\left(1-\frac{1}{\mathrm{e}}\right)|\bar{\Gamma}|$. Let $B$ be a subset of the set $A X$ such that $\bar{B}=\overline{A X}$. So $|B| \geq\left(1-\frac{1}{\mathrm{e}}\right)|\bar{\Gamma}|$ and $|A X|-|B| \leq|A||X|-\left(1-\frac{1}{\mathrm{e}}\right)|\bar{\Gamma}|$. Thus we obtain that

$$
\begin{aligned}
\left|\partial_{S}(B)\right| & \leq\left|\partial_{S}(A X)\right|+|S|(|A X|-|B|) \leq|S||A||X|(\delta+1)-\left(1-\frac{1}{\mathrm{e}}\right)|\bar{\Gamma}| \\
& \leq|S||\bar{\Gamma}|\left(1.1 \cdot \delta+0.1+\frac{1}{\mathrm{e}}\right) \leq|S| \frac{|B|}{1-\frac{1}{\mathrm{e}}}\left(1.1 \cdot \delta+0.1+\frac{1}{\mathrm{e}}\right) \\
& \leq \frac{1.4}{\mathrm{e}-1}|S||B| .
\end{aligned}
$$

We may add some $|\bar{\Gamma}|-|B|$ elements to $B$ and obtain a transversal $T$ for $\Gamma_{j}$ in $\Gamma$. Then

$$
\begin{aligned}
\left|\partial_{S}(T)\right| & \leq\left|\partial_{S}(B)\right|+|S|(|T|-|B|) \leq|S||B|\left(\frac{1.4}{\mathrm{e}-1}-1\right)+|S||T| \\
& \leq|S||T|\left(1-\left(1-\frac{1.4}{\mathrm{e}-1}\right)\left(1-\frac{1}{\mathrm{e}}\right)\right)=\frac{2.4}{\mathrm{e}}|S||T| .
\end{aligned}
$$

Thus, if we put $c=\frac{2.4}{\mathrm{e}}$ we obtain that $T$ is a $c$-invariant.

Step 2. Now suppose that for some $\sigma$ there is $k \in \mathbb{N}$ and a $\sigma$-invariant transversal $T_{1}$ of $\Gamma_{k}$ in $\Gamma$. Using the previous step we will show that there exists $l \geq k$ and a $c \sigma$-transversal $T$ for $\Gamma_{l}$ in $\Gamma$.

If $g \in \Gamma$ denote by $\tilde{g}$ the unique element from $T_{1}$ such that $g \Gamma_{k}=\tilde{g} \Gamma_{k}$. Denote by $S_{1}$ the multiset $\left\{(\tilde{s t})^{-1} s t \mid(t, s t) \in \partial_{S}\left(T_{1}\right)\right\}$. Then $S_{1}$ is a generating multiset of $\Gamma_{k}$. By the previous step, there is $l \geq k$ and a transversal $T_{2}$ for $\Gamma_{k}$ in $\Gamma_{l}$ which is $c$-invariant with respect to $S_{1}$. Put $T=T_{1} T_{2}$. Let $s \in S, t_{1} \in T_{1}$ and $t_{2} \in T_{2}$. Then for the pair $\left(t_{1} t_{2}, s t_{1} t_{2}\right)$ to be in $\partial_{S}(T)$ it is necessary that $\left(t_{1}, s t_{1}\right) \in \partial_{S}\left(T_{1}\right)$ holds together with $\left(t_{2},\left(\widetilde{s t}_{1}\right)^{-1} s t_{1} t_{2}\right) \in \partial_{S_{1}}\left(T_{2}\right)$.

Hence

$$
\left|\partial_{S}(T)\right|=\left|\partial_{S_{1}}\left(T_{2}\right)\right| \leq c\left|S_{1}\right|\left|T_{2}\right| \leq c \sigma|S|\left|T_{1}\right|\left|T_{2}\right|=c \sigma|S||T| .
$$

Iterating this process we find for any $k \in \mathbb{N}$ a transversal $T$ to some $\Gamma_{j}$ which is $c^{k}$-invariant with respect to $S$ and Theorem 6 is proved.

Now Theorem 5 follows from Theorem 6 trivially by noting that the Schreier set $\left\{(\tilde{s t})^{-1} s t \mid(t, s t) \in \partial_{S}(T)\right\}$ for the transversal $T$ from Theorem 6 is a generating set for $\Gamma_{k}$ of size $\left|\partial_{S}(T)\right| \leq \varepsilon\left|\Gamma: \Gamma_{k}\right||S|$. 


\section{Groups with a soluble normal subgroup}

In this section we prove Theorem 4 . We start with two preliminary results.

Proposition 15. Let $\Gamma$ be a group which has a sequence of finite normal subgroups $A_{i}$ such that $\left|A_{i}\right| \rightarrow \infty$. Then $\operatorname{RG}\left(\Gamma,\left(\Gamma_{j}\right)\right)=0$ for any chain $\left(\Gamma_{j}\right)$ in $\Gamma$ with trivial intersection.

Proof. Let $d=d(\Gamma)$. Suppose $\left|A_{i}\right|=a_{i}$ and let $H=\Gamma_{j}$ be a member of the chain $\left(\Gamma_{i}\right)$ of $\Gamma$, such that $A_{i} \cap \Gamma_{j}=1$. If $\left|\Gamma: A_{i} H\right|=a$ then $|\Gamma: H|=a a_{i}$ and $A_{i} H$ can be generated by $(d-1) a+1 \leq d a$ elements. Therefore same holds for its homomorphic image $H$, and so $r\left(\Gamma, \Gamma_{j}\right) \leq \frac{d}{a_{i}}$. Since $a_{i} \rightarrow \infty$ as $i$ increases we get $\operatorname{RG}\left(\Gamma,\left(\Gamma_{i}\right)\right)=0$.

Lemma 16. Let $\Gamma$ be a $d$-generated group and $N$ a $\mathbb{Z} \Gamma$-module generated by $t$ elements as a module over $\mathbb{Z} \Gamma$. Suppose that $N_{0} \leq N$ is a $\mathbb{Z} \Gamma$-submodule of index $b$. Then $N_{0}$ can be generated by at most $t+(2 d+1) \log b$ elements as a $\mathbb{Z} \Gamma$-module.

We postpone the proof to the end of this section and move to

Proof of Theorem 4. Suppose $\left(\Gamma_{i}\right)$ is a chain with trivial intersection in a group $\Gamma$ which has an infinite soluble normal subgroup, call it $S$. Consider the last infinite term $A$ of the derived series of $S$. Then $A^{\prime}$ is finite and it is easy to see that $\operatorname{RG}\left(\Gamma, \Gamma_{i}\right)=0$ if and only if $\operatorname{RG}\left(\Delta,\left(\Delta_{i}\right)\right)=0$ where $\Delta_{i}=\Gamma_{i} A^{\prime} / A^{\prime}$ and $\Delta=\Gamma / A^{\prime}$.

Hence by considering $\Gamma / A^{\prime}$ in place of $\Gamma$ we may assume that $A^{\prime}=1$ and $\Gamma$ has an infinite abelian normal subgroup $A$.

Case 1. Suppose that the normal closure of every element of $A$ is finite. Then we can find a sequence $A_{i}$ of finite subgroups $A_{i}$ of $A$, all normal in $\Gamma$, such that $\left|A_{i}\right| \rightarrow \infty$. By Proposition 15 we are done.

Case 2. Suppose that $A$ has an element whose normal closure in $\Gamma$ is infinite. Without loss of generality we may assume that $A$ is a principal, i.e., 1-generated $\Gamma$-module. For each $j \in \mathbb{N}$ put $M_{j}=A \cap \Gamma_{j}$ and $\bar{\Gamma}_{j}=A \Gamma_{j}$. Suppose that $\left|\Gamma: \bar{\Gamma}_{j}\right|=a_{j}$ and $\left|A: M_{j}\right|=b_{j}$, so that $\left|\Gamma: \Gamma_{j}\right|=a_{j} b_{j}$. Let $d=d(\Gamma)$. It follows that $d\left(\bar{\Gamma}_{j}\right)<a_{j} d$ and therefore the same is true for $\bar{\Gamma}_{j} \simeq \Gamma_{j} / M_{j}$. Let $\Gamma_{j}=\left\langle h_{1}, \ldots, h_{p}\right\rangle M_{j}$ with $p<a_{j} d$.

Since $A$ is a principal $\Gamma$-module it is $a_{j}$-generated as a $\Gamma_{j}$-module. Now $M_{j}$ is a $\Gamma_{j}$ invariant subgroup of $A$ of index $b_{j}$. By Lemma $16 M_{j}$ is generated by at most $a_{j}+\left(2 d\left(\bar{\Gamma}_{j}\right)+1\right) \log b_{j}$ elements as a module over $\bar{\Gamma}_{j}$. Let $M_{j}=\left\langle n_{1}, \ldots, n_{q}\right\rangle^{\Gamma_{j}}$ with $q \leq a_{j}+\left(2 d a_{j}+1\right) \log b_{j}$.

We claim that $\left\langle h_{1}, \ldots, h_{p}, n_{1}, \ldots, n_{q}\right\rangle=\Gamma_{j}$. Indeed, $A$ acts trivially by conjugation on $M_{j}$ and $\left\langle h_{1}, \ldots, h_{p}\right\rangle A=\Gamma_{j}$. Therefore $M_{j}=\left\langle n_{1}, \ldots, n_{q}\right\rangle^{\bar{\Gamma}_{j}}=$ 
$\left\langle n_{1}, \ldots, n_{q}\right\rangle^{\left\langle h_{1}, \ldots, h_{p}\right\rangle}$, while $\Gamma_{j}=\left\langle h_{1}, \ldots, h_{p}\right\rangle M_{j}$. It follows that $d\left(\Gamma_{j}\right) \leq p+q$ and hence

$$
\frac{d\left(\Gamma_{j}\right)-1}{\left|\Gamma: \Gamma_{j}\right|} \leq \frac{p+q}{a_{j} b_{j}}<\frac{d}{b_{j}}+\frac{1+(2 d+1) \log b_{j}}{b_{j}} .
$$

Since $\bigcap_{j} \Gamma_{j}=\{1\}$ the index $b_{j}=\left|A: A \cap \Gamma_{j}\right| \rightarrow \infty$ with $j$ and so the right hand side tends to 0 .

This completes Case 2 and the proof of Theorem 4.

Proof of Lemma 16. First we shall prove the lemma in the special case when $t=1$, i.e. $N$ is a principal $\mathbb{Z} \Gamma$ module. Without loss of generality we can assume that $N=\mathbb{Z} \Gamma$. Let $\left\langle g_{1}, \ldots, g_{d}\right\rangle=\Gamma$.

Pick $f_{1}, \ldots, f_{s} \in N$ whose images in $N / N_{0}$ generate it as an abelian group and such that $s \leq \log b$. Let $K=\left\langle f_{1}, \ldots, f_{s}\right\rangle \leq N$ and let $K_{0}=K \cap N_{0}$. It follows that the abelian group $K_{0}$ can be generated by at most $s$ elements, say $c_{1}, \ldots, c_{s} \in N$. Since $N=K+N_{0}$ there exists $e \in K$ such that $1-e \in N_{0}$. Moreover, for each pair of indices $(i, j)$ with $1 \leq i \leq d, 1 \leq j \leq s$ and each $\epsilon \in\{ \pm 1\}$ there exists $e_{i, j, \epsilon} \in K$ such that

$$
f_{j} \cdot g_{i}^{\epsilon}-e_{i, j, \epsilon} \in N_{0} .
$$

Let $M$ be the $\mathbb{Z} \Gamma$-submodule of $N$ generated by the $1+(2 d+1) s$ elements $1-e$, $\left\{c_{k}\right\}$, and $\left\{f_{j} \cdot g_{i}^{\epsilon}-e_{i, j, \epsilon}\right\}$. We claim that $M=N_{0}$.

It is clear that $M \leq N_{0}$. For the opposite direction we first show that $N=K+M$. It is enough to prove that each $g \in \Gamma \subset \mathbb{Z} \Gamma=N$ is in $K+M$. When $g=1$ we have $1=e+(1-e)$ with $e \in K$ and $1-e \in M$. Observe that the elements (2) of $M$ give $K \cdot g_{i}^{\epsilon} \subseteq K+M$. Now $g=1 \cdot g \equiv e \cdot g \bmod M$ and use induction on the length of the shortest expression of $g$ as a product of $g_{i}^{ \pm 1}$.

Hence $N=K+M$ and so $N_{0}=K_{0}+M$. But $K_{0}=\left\langle c_{1}, \ldots, c_{s}\right\rangle \leq M$, hence $K_{0} \leq M$ and $N_{0}=M$ as claimed. This proves case $t=1$ of Lemma 16.

Now we can prove the general case by induction on $t$. Take a submodule $K<M$ which is $t-1$ generated and $M / K$ is a principal $\mathbb{Z} \Gamma$-module. Let $b_{1}$ be index of $N_{0} \cap K$ in $K$ and $b_{2}$ be the index of $N_{0} K$ in $N$. Then $b_{1} b_{2}=b$ and we may assume that $K \cap N_{0}$ is $t-1+(1+2 d) \log b_{1}$ generated and $N_{0} / N_{0} \cap K \simeq N_{0} K / K$ is $1+(1+2 d) \log b_{2}$ generated as $\mathbb{Z} \Gamma$ modules. Now since $N_{0}$ is an extension of $N_{0} \cap K$ by $N /\left(N_{0} \cap K\right)$ it is generated by at most

$$
t-1+(1+2 d) \log b_{1}+1+(1+2 d) \log b_{2}=t+(1+2 d) \log b
$$

elements. Lemma 16 follows.

\section{Virtually free groups and free products with amalgamation}

We start by analyzing when $r\left(\Gamma, \Gamma_{i}\right)$ stabilizes. 
Proof of Theorem 7. Let $r_{i}=r\left(\Gamma, \Gamma_{i}\right)$.

i). Assume that $\Gamma$ is virtually free. Then by Bass-Serre theory $\Gamma$ has only finitely many conjugacy classes of finite subgroups. As the groups $\Gamma_{i}$ are normal and $\bigcap_{i} \Gamma_{i}=\{1\}$ there is $n \in \mathbb{N}$ such that $\Gamma_{n}$ is torsion free. Now by the Stallings theorem [13] every torsion free virtually free group is free, hence $\Gamma_{n}$ is free and then obviously $r_{i}=r_{n}$ for $i \geq n$.

ii). Now assume that $r_{i}=r_{n}$ for all $i \geq n$. By considering $\Gamma_{n}$ in place of $\Gamma$ we may assume that $n=0$ and $r_{i}=d(\Gamma)-1$ for all $i \geq 0$. We show that $\Gamma$ is free.

Let $d=d(\Gamma)$ and let $\Gamma=\langle S\rangle$, where $S=\left\{s_{1}, \ldots, s_{d}\right\}$. Consider the free group $F$ on $d$ free generators $x_{1}, \ldots, x_{d}$ and the epimorphism $f: F \rightarrow \Gamma$ given by $f\left(x_{i}\right)=s_{i}$. We claim that $f$ is an isomorphism. Assume not. Let $w=$ $x_{i_{k}}^{\epsilon_{k}} x_{i_{k-1}}^{\epsilon_{k-1}} \ldots x_{i_{1}}^{\epsilon_{1}}$ be the shortest nontrivial word in $\operatorname{ker} f$.

Consider the segments $w_{j}=x_{i_{j}}^{\epsilon_{j}} x_{i_{j-1}}^{\epsilon_{j-1}} \ldots x_{i_{1}}^{\epsilon_{1}}$ for $1 \leq j \leq k$. The choice of $w$ gives that the $k$ elements $f\left(w_{j}\right), j=1, \ldots, k$ of $\Gamma$ are all different and therefore there exists an integer $m$ such that $f\left(w_{j}\right)^{-1} f\left(w_{i}\right) \notin \Gamma_{m}$ for all $1 \leq i \neq j \leq m$. Put $H=\Gamma_{m}$, and let $\Delta=\Delta(\Gamma, H, S)$. Fix the left Schreier transversal $T$ of $H$ in $\Gamma$ corresponding to a maximal tree $\mathcal{T}$. Then by the choice of $\Gamma_{m}$ we may write $f(w)$ as a product of elements from $\{T(e), e \in E(\Delta)\}, f(w)=T\left(e_{i_{k}}\right)^{\epsilon_{k}} \ldots T\left(e_{i_{1}}\right)^{\epsilon_{1}}$, in such way that all $e_{i_{j}}$ are different. There exist $j$ such that $e_{i_{j}}$ is not in $E(\mathcal{T})$, whence we obtain that $T\left(e_{i_{j}}\right)$ may be expressed in terms of other generators $\{T(e), e \in$ $E(\Delta) \backslash E(\mathcal{T})\}$ of $H$. It follows that $d\left(\Gamma_{m}\right)<(d-1)\left|\Gamma: \Gamma_{m}\right|+1$ and so $r_{m}<d-1$, a contradiction. Hence $\Gamma$ is free.

Now we discuss the rank gradient of free products with amalgamation.

Proof of Proposition 8. Let $N$ be a normal subgroup of index $n$ in $\Gamma=G_{1} \star G_{2}$ and denote $N_{j}=N \cap G_{j}$. Suppose that $\left|G_{j}: N_{j}\right|=\left|G_{j} N: N\right|=k_{j}$. The Bass-Serre theory gives us the structure of $N$ : it is a free product of $n / k_{1}$ copies of $N_{1}$ with $n / k_{2}$ copies of $N_{2}$ and a free group of rank $n-\frac{n}{k_{1}}-\frac{n}{k_{2}}+1$. (See the proof of Proposition 9 below where a similar computation is given in the case of an amalgam.) By the Grushko-Neumann theorem (see Proposition 3.7 in [8]) we have

$$
d(N)=\frac{n}{k_{1}} d\left(N_{1}\right)+\frac{n}{k_{2}} d\left(N_{2}\right)+n-\frac{n}{k_{1}}-\frac{n}{k_{2}}+1 .
$$

Hence

$$
\frac{d(N)-1}{|\Gamma: N|}=\frac{d\left(N_{1}\right)-1}{\left|G_{1}: N_{1}\right|}+\frac{d\left(N_{2}\right)-1}{\left|G_{2}: N_{2}\right|}+1 .
$$

Taking the group $N$ to range over the normal chain $\left(\Gamma_{i}\right)$ Proposition 8 follows.

Proof of Proposition 9. Denote by $A$ the intersection of $G_{1}$ and $G_{2}$. Let $\widetilde{\Gamma}=G_{1} \star_{A}$ $G_{2}$ and let $\pi$ be the natural projection $\pi: \widetilde{\Gamma} \rightarrow \Gamma$. By a slight abuse of notation we shall identify the groups $G_{1}, G_{2}$ and $A$ with their preimages under $\pi$. 
Let $N$ be a normal subgroup of index $n$ in $\Gamma$ and take $\tilde{N}=\pi^{-1}(N)$. Then $\tilde{N} \cap G_{j} \leq \widetilde{\Gamma}$ is isomorphic to $N \cap G_{j} \leq \Gamma$ under $\pi$. Moreover $d(N) \leq d(\tilde{N})$ and $|\Gamma: N|=|\widetilde{\Gamma}: \widetilde{N}|$ so it is enough to obtain an upper bound for $(d(\widetilde{N})-1) /|\widetilde{\Gamma}: \widetilde{N}|$.

We use the techniques of Bass-Serre theory, (as explained in [11] for example). The group $\widetilde{\Gamma}$ acts on a tree $T$ with fundamental domain an edge $E \subset T$ with vertices $X_{1}$ and $X_{2}$ such that $G_{j}=\operatorname{Stab}_{\Gamma}\left(X_{j}\right)$ and $A=\operatorname{Stab}_{\Gamma}(E)$.

Let $a=[\tilde{N} A: \tilde{N}]=|A:(N \cap A)|$ and $k_{j}=\left[\tilde{N} G_{j}: \tilde{N}\right]=\left[G_{j}:\left(G_{j} \cap \tilde{N}\right)\right]$ for $j=1,2$. Then $\tilde{N}$ is the fundamental group of the graph of groups $\mathscr{E}=\tilde{N} \backslash T$. The graph $G$ has $\left[\widetilde{\Gamma}: G_{j} \tilde{N}\right]=n / k_{j}$ vertices of type $X_{j}$ for $j=1,2$, and it has $n / a=[\tilde{\Gamma}: A \tilde{N}]$ number of edges. The stabilizers of vertices of type $X_{j}$ in $\mathscr{E}$ are isomorphic to $\tilde{N} \cap G_{j} \simeq N \cap G_{j}(j=1,2)$, and all the edge stabilizers are isomorphic to $\tilde{N} \cap A \simeq N \cap A$.

Now by the presentation of the fundamental group of a graph of groups in b), page 42 of [11], it follows that $\tilde{N}$ is generated by the stabilizers of the vertices of $\mathscr{E}$ together with elements $t_{y}$ for each edge of $\mathcal{E}$ which lies outside some chosen maximal spanning tree of $\mathscr{G}$. The total number of vertices of $\mathscr{E}$ is $n / k_{1}+n / k_{2}$ and the number of edges is $n / a$. It follows that

$$
d(\tilde{N}) \leq \frac{n}{k_{1}} d\left(\tilde{N} \cap G_{1}\right)+\frac{n}{k_{2}} d\left(\tilde{N} \cap G_{2}\right)+\frac{n}{a}-\frac{n}{k_{1}}-\frac{n}{k_{2}}+1,
$$

and hence

$$
\frac{d(N)-1}{[\Gamma: N]} \leq \frac{d(\tilde{N})-1}{[\widetilde{\Gamma}: \tilde{N}]} \leq \frac{d\left(N^{(1)}\right)-1}{\left[\Gamma_{1}: N^{(1)}\right]}+\frac{d\left(N^{(2)}\right)-1}{\left[\Gamma_{2}: N^{(2)}\right]}+\frac{1}{a} .
$$

where for $j=1,2$ we denote $N^{(j)}=N \cap \Gamma_{j} \simeq \tilde{N} \cap \Gamma_{j}$.

Now take the subgroup $N$ to range over the normal chain $\left(\Gamma_{i}\right)$ of $\Gamma$. Then $\left(\Gamma_{i}^{(1)}\right)$ and $\left(\Gamma_{i}^{(2)}\right)$ are normal chains in $G_{1}$ and $G_{2}$, respectively, and the numbers $a=\mid A$ : $\left(A \cap \Gamma_{i}\right) \mid$ tend to infinity with $i$. The proposition follows.

\section{Lück approximation and boundedly generated groups}

Lück approximation gives a fast proof for Proposition 11.

Proof of Proposition 11. Let $\Gamma=\left\langle g_{1}\right\rangle \cdot\left\langle g_{2}\right\rangle \ldots\left\langle g_{t}\right\rangle$ and let $\left(\Gamma_{i}\right)$ be a normal chain in $\Gamma$ with trivial intersection. Let $K_{i}=\Gamma_{i}^{\prime} \Gamma_{i}^{2}$, let $G_{i}=\Gamma / \Gamma_{i}$ and let $H_{i}=\Gamma / K_{i}$ $(i \geq 0)$. Since $\Gamma$ is infinite, $\left|G_{i}\right|$ tends to infinity with $i$.

Now $\Gamma_{i} / K_{i}$ is an elementary abelian 2-group of $\operatorname{rank} r_{i}$ and the exponent $\exp \left(H_{i}\right)$ of $H_{i}$ is at most $2\left|G_{i}\right|$. Hence

$$
\left|G_{i}\right| 2^{r_{i}}=\left|H_{i}\right| \leq \exp \left(H_{i}\right)^{t} \leq\left(2\left|G_{i}\right|\right)^{t}
$$


and so $r_{i} \leq t+(t-1) \log _{2}\left|G_{i}\right|$. Let $d_{i}$ denote the first Betti number of $\Gamma_{i}$. Then, using a theorem of Lück [7], we have

$$
\beta_{1}^{2}(\Gamma)=\lim _{n \rightarrow \infty} \frac{d_{n}}{\left|G_{n}\right|} \leq \lim _{n \rightarrow \infty} \frac{r_{n}}{\left|G_{n}\right|} \leq \lim _{n \rightarrow \infty} \frac{t+(t-1) \log _{2}\left|G_{n}\right|}{\left|G_{n}\right|}=0 .
$$

The proposition holds.

We finish the paper by providing a simple proof of a result of Gabor Elek on Lück approximation over arbitrary field for amenable groups.

Theorem 17. Let $K$ be a field and $\Gamma$ a finitely generated amenable group and let ( $\left.\Gamma_{i}\right)$ be a normal chain with trivial intersection in $\Gamma$. Suppose $A \in \mathbb{M}_{n \times m}(K[\Gamma])$ is a matrix over the group algebra $K[\Gamma]$ and let $A_{i}$ be the image of $A$ in $\mathbb{M}_{n \times m}\left(K\left[\Gamma / \Gamma_{i}\right]\right)$ under the quotient map $\pi_{i}: \Gamma \rightarrow \Gamma / \Gamma_{i}$. Then

$$
\lim _{i \rightarrow \infty} \frac{\operatorname{dim}_{K} \operatorname{ker} A_{i}}{\left|\Gamma: \Gamma_{i}\right|}
$$

exists and does not depend on the choice of the chain $\left(\Gamma_{i}\right)$.

It is an important question whether one can omit the amenability assumption in this result for $K=\mathbb{F}_{p}$. The proof of the theorem uses the Ornstein-Weiss lemma proved in [9]. Our exposition of this result is based on a paper of Gromov (see [5], p. 336).

Lemma 18 (Ornstein-Weiss). Let $\Gamma$ be a finitely generated amenable group. Let $h(\Omega)$ be a positive function defined on finite subsets $\Omega$ of $\Gamma$ such that

1. $h$ is subadditive, i.e.

$$
h\left(\Omega_{1} \cup \Omega_{2}\right) \leq h\left(\Omega_{1}\right)+h\left(\Omega_{2}\right)
$$

for all pairs of finite subsets $\Omega_{1}$ and $\Omega_{2}$ in $\Gamma$;

2. $h$ is right $\Gamma$-invariant under $\Gamma$,

$$
h(\Omega \gamma)=h(\Omega), \text { for all } \gamma \in \Gamma
$$

Then the limit

$$
\lim _{i \rightarrow \infty} h\left(\Omega_{i}\right) /\left|\Omega_{i}\right|
$$

exists for every Folner sequence $\Omega_{i} \subset \Gamma$. Moreover, this limit does not depend on the choice of the Folner sequence $\left(\Omega_{i}\right)$. 
Proof of Theorem 17. For any $c=\sum_{\gamma \in \Gamma} c_{\gamma} \gamma \in K[\Gamma]$ let $\operatorname{supp}(\gamma)$ be the set $\{\gamma$ : $\left.c_{\gamma} \neq 0\right\}$. Let $\Omega$ be a finite subset of $\Gamma$. By $\boldsymbol{b} \in K[\Gamma]^{m}$ we denote the column vector $\left(b_{1}, \ldots, b_{m}\right)^{T}$ with entries $b_{i} \in K[\Gamma]$. We denote the function $h$ as follows

$$
h(\Omega)=\operatorname{dim}_{K}\left\{A \boldsymbol{b} \in(K[\Gamma])^{n}: \operatorname{supp}\left(b_{i}\right) \subset \Omega\right\} \text {. }
$$

It is clear that $h$ is subadditive and right invariant. Thus, by the Ornstein-Weiss lemma, there exists $H=\lim _{i \rightarrow \infty} h\left(\Omega_{i}\right) /\left|\Omega_{i}\right|$ for every Følner sequences $\Omega_{i} \subset \Gamma$. We will see now that the limit from Theorem 17 is equal to $m-H$.

Since $\operatorname{dim}_{K} \operatorname{ker} A_{i} /\left|\Gamma: \Gamma_{i}\right|$ is bounded, in order to prove that the sequence $\operatorname{dim}_{K} \operatorname{ker} A_{i} /\left|\Gamma: \Gamma_{i}\right|$ tends to $m-H$, it is enough to show the limit of any Cauchy subsequence of $\operatorname{dim}_{K} \operatorname{ker} A_{i} /\left|\Gamma: \Gamma_{i}\right|$ is $m-H$. Thus, without loss of generality we may assume that $\lim _{i \rightarrow \infty} \operatorname{dim}_{K} \operatorname{ker} A_{i} /\left|\Gamma: \Gamma_{i}\right|$ exists and we want to show that it is equal to $m-H$.

Let $S$ be a generating set of $\Gamma$ containing the supports of all the entries of the matrix $A$. By Theorem 6 , for any $\varepsilon>0$ there exist $j$ and a transversal $T_{j}$ of $\Gamma_{j}$ in $\Gamma$ such that $\left|\partial_{S} T_{j}\right| \leq \varepsilon\left|T_{j}\right|$. Hence

$h\left(T_{j}\right) \geq \operatorname{dim}_{K} \operatorname{Im} A_{j} \geq \operatorname{dim}_{K}\left\{\boldsymbol{a}=A \boldsymbol{b} \mid \operatorname{supp}\left(b_{i}\right), \operatorname{supp}\left(a_{i}\right) \subset T_{j}\right\} \geq h\left(T_{j}\right)-\varepsilon\left|T_{j}\right|$

where $\boldsymbol{a}=\left(a_{1}, \ldots, a_{m}\right)^{T}$ is a column vector in $K[\Gamma]^{m}$. This implies that

$$
\lim _{i \rightarrow \infty} \operatorname{dim}_{K} \operatorname{ker} A_{i} /\left|\Gamma: \Gamma_{i}\right|=m-H
$$

Acknowledgement. The authors wish to thank Laci Pyber for helpful comments on Lemma 14 and Alessandro Carderi for spotting some typos.

\section{References}

[1] M. Abért and N. Nikolov, Rank gradient, cost of groups and the rank vs. Heegaard genus conjecture. J. Eur. Math. Soc. (JEMS), to appear. arXiv:math/0701361

[2] M. Abért and G. Elek, Dynamical properties of profinite actions. Preprint 2010. arXiv: 1005.3188

[3] M. E. B. Bekka and A. Valette, Group cohomology, harmonic functions and the first $L^{2}$-Betti number. Potential Anal. 6 (1997), 313-326. Zbl 0882.22013 MR 1452785

[4] R. I. Grigorchuk, V. V. Nekrashevich, and V. I. Sushchanskiǔ, Automata, dynamical systems, and groups. Trudy Mat. Inst. Steklov. 231 (2000) , 134-214; English transl. Proc. Steklov Inst. Mat. 231 (2000), 134-214. Zbl 1155.37311 MR 1841755

[5] M. Gromov, Topological invariants of dynamical systems and spaces of holomorphic maps. I. Math. Phys. Anal. Geom. 2 (1999), 323-415. Zbl 1160.37322 MR 1742309

[6] M. Lackenby, Expanders, rank and graphs of groups. Israel J. Math. 146 (2005), 357-370. Zbl 1066.22008 MR 2151608 
[7] W. Lück, $L_{2}$-invariants: theory and applications to geometry and $K$-theory. Ergeb. Math. Grenzgeb. (3) 44, Springer-Verlag, Berlin 2002. Zbl 1009.55001 MR 1926649

[8] R. C. Lyndon and P. E. Schupp, Combinatorial group theory. Ergeb. Math. Grenzgeb. 89, Springer-Verlag, Berlin 1977. Zbl 0368.20023 MR 0577064

[9] D. S. Ornstein and B. Weiss, Entropy and isomorphism theorems for actions of amenable groups. J. Analyse Math. 48 (1987), 1-141. Zbl 0637.28015 MR 910005

[10] K. Schmidt, Amenability, Kazhdan's property $T$, strong ergodicity and invariant means for ergodic group-actions. Ergodic Theory Dynam. Systems 1 (1981), 223-236. Zbl 0485.28019 MR 661821

[11] J.-P. Serre, Trees. Springer-Verlag, Berlin 1980. Zbl 0548.20018 MR 0607504

[12] R. Sharma and T. N. Venkataramana, Generations for arithmetic groups. Geom. Dedicata 114 (2005), 103-146. Zbl 1112.20044 MR 2174097

[13] J. R. Stallings, On torsion-free groups with infinitely many ends. Ann. of Math. (2) 88 (1968), 312-334. Zbl 0238.20036 MR 0228573

[14] O. I. Tavgen', Bounded generation of Chevalley groups over rings of $S$-integer algebraic numbers. Izv. Akad. Nauk SSSR Ser. Mat. 54 (1990), 97-122; English transl. Math. USSRIzv. 36 (1991), 101-128. Zbl 0697.20032 MR 1044049

[15] B. Weiss, Monotileable amenable groups. In Topology, ergodic theory, real algebraic geometry, Amer. Math. Soc. Transl. Ser. (2) 202, Amer. Math. Soc., Providence, RI, 2001, 257-262. Zbl 0982.22004 MR 1819193

Received May 12, 2009; revised April 23, 2010

M. Abért, Department of Mathematics, Alfréd Rényi Institute of Mathematics, Hungarian Academy of Sciences, P.O. Box 127, 1364 Budapest, Hungary

E-mail: abert@renyi.hu

A. Jaikin-Zapirain, Departamento de Matemáticas, Universidad Autónoma de Madrid and Instituto de Ciencias Matemáticas, CSIC-UAM-UC3M-UCM, Madrid, Spain

E-mail: andrei.jaikin@uam.es

N. Nikolov, Department of Mathematics, Imperial College London, SW7 2AZ, United Kingdom

E-mail: n.nikolov@imperial.ac.uk 